The following scientific article was officially published in the Proceedings of the IEEE International Symposium on Biomedical Imaging. This article's citation is as follows:

Seoud, L., Adankon, M.M., Labelle, H., Dansereau, J. and Cheriet, F., "Prediction of scoliosis curve type based on the analysis of trunk surface topography." In 2010 IEEE International Symposium on Biomedical Imaging: From Nano to Macro (2010), pp. 408411.

doi: $\underline{\text { http://dx.doi.org/10.1109/ISBI.2010.5490322 }}$

The manuscript, as accepted by the publisher, is reproduced in the following pages.

(C) 2010 IEEE. Personal use of this material is permitted. Permission from IEEE must be obtained for all other users, including reprinting/republishing this material for advertising or promotional purposes, creating new collective works for resale or redistribution to servers or lists, or reuse of any copyrighted components of this work in other works. 


\title{
PREDICTION OF SCOLIOSIS CURVE TYPE BASED ON THE ANALYSIS OF TRUNK SURFACE TOPOGRAPHY
}

\author{
L. Seoud $^{1,2}$, M. M. Adankon ${ }^{3}$, H. Labelle ${ }^{2}$, J. Dansereau ${ }^{1,2}$, F. Cheriet $^{1,2}$ \\ (1) École Polytechnique de Montréal, Montréal, Canada \\ (2) Sainte-Justine Hospital Research Center, Montréal, Canada \\ (3) École de Technologie Supérieure, Montréal, Canada
}

\begin{abstract}
Scoliosis treatment strategy is generally chosen according to the severity and type of the spinal curve. Currently, the curve type is determined from X-rays whose acquisition can be harmful for the patient. We propose in this paper a system that can predict the scoliosis curve type based on the analysis of the surface of the trunk. The latter is acquired and reconstructed in 3D using a non invasive multi-head digitizing system. The deformity is described by the back surface rotation, measured on several cross-sections of the trunk. A classifier composed of three support vector machines was trained and tested using the data of 97 patients with scoliosis. A prediction rate of $72.2 \%$ was obtained, showing that the use of the trunk surface for a high-level scoliosis classification is feasible and promising.
\end{abstract}

Index Terms - Pattern classification, Scoliosis, Surface topography.

\section{INTRODUCTION}

Scoliosis is a three-dimensional deformity of the spine and ribcage that affects the general appearance of the trunk. Among the external manifestations of scoliosis, the rib hump, a protuberance that appears on the back, constitutes the most disturbing aspect of the deformity for the patient. Clinically, the evaluation of scoliosis relies essentially on two radiographs, frontal and lateral, of the patient's trunk, in upright position. Clinicians can then visualize the spinal deformity and determine its type and severity. For the most severe cases of scoliosis, a surgical treatment is generally undertaken. The choice of surgical strategy is based on the type and severity of the deformity.

Even though the radiographic evaluation of scoliosis is standard clinical practice, it has several limitations. First, the $\mathrm{X}$-rays acquisition is invasive and its cumulation throughout the follow-up can be harmful for patients [1]. For this reason, its frequency is limited to every 6 to 8 months, which represents a long interval in the case of progressive scoliosis. Second, only the internal deformities can be evaluated from the X-rays while the patients' main concern is their external appearance. Finally, barring a 3D reconstruction of the spine by stereo radiography, the information contained in an X-ray is bi-dimensional and thus not sufficient to fully evaluate a complex 3D deformity.

To overcome these limitations, many optical non invasive surface measurement systems have been developed over the last 30 years. This approach can provide a 3D reconstruction of the back or of the whole trunk, with more or less resolution and accuracy depending on the acquisition system used. The non invasiveness of the acquisition allows for a more frequent evaluation. The objective is to reduce, and not to substitute, the radiographic evaluation. Currently, the main challenge is to determine the most relevant features on the surface of the trunk for scoliosis evaluation. Therefore, few groups have tried to predict the severity of scoliosis [2-4] or the 3D shape of the spine [5] from the evaluation of the surface of the back or of the trunk, using statistical methods [4] or machine learning techniques [2, 5]. However, to our knowledge, the prediction of scoliosis curve type has never been attempted, even though, in surgical cases, the treatment strategy is chosen according to the number and the location of the scoliosis spinal curves.

The objective of this paper is to predict the scoliosis curve type from the analysis of the trunk surface, acquired and reconstructed in 3D using a non invasive system. Specifically, we aim to identify and extract relevant features on the trunk, to build a classifier based on these features and to evaluate its performance in terms of prediction.

\section{MATERIALS AND METHODS}

\subsection{Data acquisition}

Currently, at Sainte-Justine University Hospital Center (SJUHC) in Montreal (Canada), the acquisition of the surface topography of the trunk is part of the routine evaluation of patients with scoliosis. The acquisition system is composed of 4 optical digitizers (InSpeck Inc., Montréal, Canada), each one comprised of a color CCD camera and a structured light projector. The acquisition process is the same for each scanner. Four fringe patterns, obtained by phase-shifting a set of light fringes, are successively projected onto the surface. Based on the four resulting images, the system computes, by triangulation, the depth of each surface point relative to the reference plane of the 


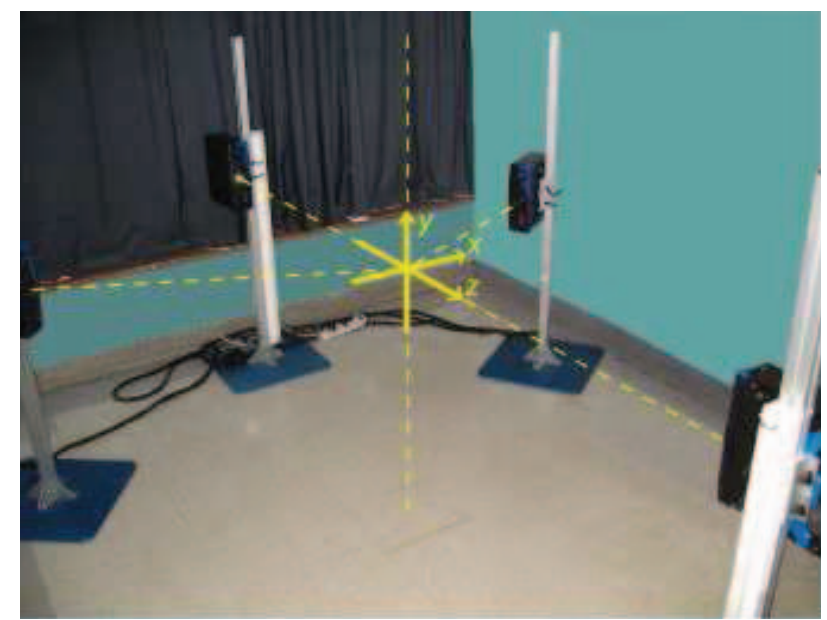

Fig. 1 - InSpeck digitizers' configuration at SJUHC.

digitizer. A fifth image, with no fringes, acquires the texture of the surface which is then mapped onto the 3D reconstruction.

For the reconstruction of the whole trunk, 4 scanners are placed around the patient (on the front, on the back and at $\pm 60^{\circ}$ laterally in front of the patient) (Fig. 1). Each digitizer reconstructs a portion of the trunk. During the acquisition, which lasts approximately 4 seconds, the patient stands still in the upright position with the arms slightly abducted in order not to obstruct the lateral scanners' fields of view. Based on a multi-head calibration of the system that computes the rigid transformations between the digitizers, the 4 portions of the trunk are registered and merged. The resulting mesh is constituted of 40,000 to 70,000 nodes, depending on the size of the patient.

The accuracy of this system was evaluated in [6], using markers placed on a mannequin whose coordinates were previously recorded by a computer measuring machine. The results showed a reconstruction accuracy of $1.4 \mathrm{~mm}$ over the whole torso and of $0.56 \mathrm{~mm}$ over the back.

In practice, prior to the acquisition, a nurse locates several anatomic landmarks on the trunk by palpation and places markers over them. These markers include the left and right antero-superior iliac spines (ASIS), the center of the postero-superior iliac spines (CPSIS), the vertebral prominence and the spinous processes of 2 or 3 vertebrae. These markers are used in order to compute clinical indices and to define a patient-specific 3D reference frame.

In fact, to allow comparison between the reconstructions of several patients or of the same patient at different acquisition dates, it is important to transpose the trunk reconstruction into a model-specific 3D reference frame. According to [7], this coordinate system is centered on the CPSIS, with the $\mathrm{Y}$-axis pointing vertical upward, the $\mathrm{X}$-axis parallel to the line joining the ASIS projected onto the axial plane, pointing to the right of the patient, and the Z-axis oriented toward the back of the patient.
Table I - Patients distribution among the classes

\begin{tabular}{cc}
\hline Class & $\begin{array}{c}\text { Number of } \\
\text { patients }\end{array}$ \\
\hline \hline C1 & 28 \\
C2 & 42 \\
C3 & 27 \\
\hline Total & 97 \\
\hline
\end{tabular}

\subsection{Clinical data}

This study was conducted on a cohort of 97 adolescents with scoliosis who were candidates for surgery, using acquisitions taken before surgery. Based on the radiographs of each patient, a clinical expert determined the type of the scoliosis curve according to a well known clinical classification [8]. The Lenke system distinguishes between 6 types of curves (L1 to L6). However, in the present study, because of the limited number of samples per class, we focused on a high level classification with 3 classes: thoracic major curves (union of types L1 and L2), double and triple major curves (union of types L3 and L4) and lumbar major curves (union of types L5 and L6). The distribution of the patients among the classes is illustrated in Table I.

\subsection{Features extraction}

To evaluate the scoliosis deformities on the surface of the trunk, local measurements are computed on inclined crosssections that follow the shape of the trunk. The sections extraction approach (Fig. 2), initially proposed in [9], consists of defining 3 guiding curves along the trunk : a first curve on the back that joins the vertebral prominence, two points along the back valley and the CPSIS, and two other curves on the front that join the clavicle medial extremity, the nipple, the waist cavity and the ASIS, on the left and right sides of the trunk. These curves are represented by cubic splines, the control points (the previous landmarks) of which are equally spaced along each curve. Subsequently, 100 points are sampled uniformly on each curve. At each of the 100 levels, a plane is defined by the points on the 3 curves. Its intersection with the mesh determines a trunk cross-section.

Then, a principal component analysis is performed on the coordinates of the points composing each section in order to compute its major and minor axes, from which a section-specific local axis system is defined.

In the local reference frame of each of the 100 sections, an automatic algorithm calculates the back surface rotation (BSR). This clinical measurement is related to the amplitude of the rib hump and is defined as the angle, projected onto the axial plane, between the dual tangent to the posterior portion of the section and the X-axis of the patient reference frame. The BSR can be negative or positive depending on the side of the hump. The accuracy of this measurement 

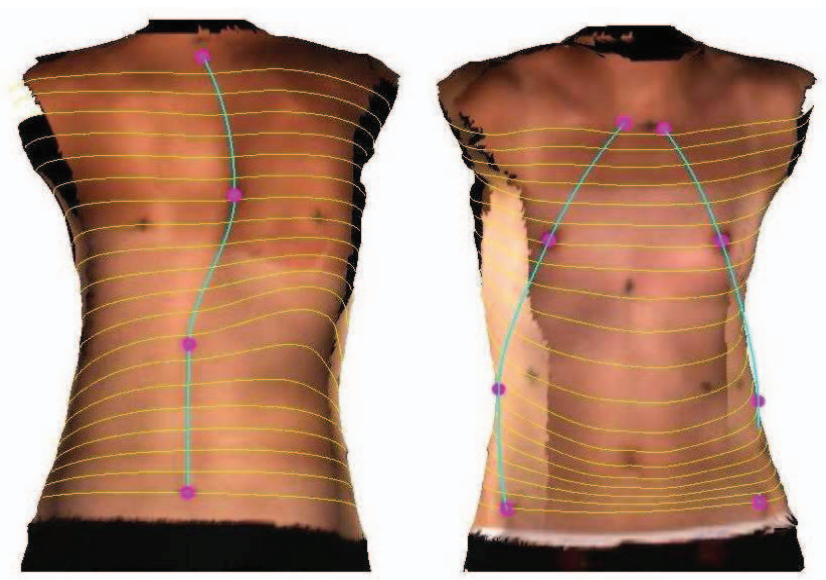

Fig. 2 - Trunk sections extraction: the three guiding curves (blue vertical lines), the anatomical landmarks (points in magenta) and 20 extracted sections (yellow horizontal lines).

computed on a $3 \mathrm{D}$ reconstruction of the trunk surface was previously evaluated at $1.4^{\circ}$ [7].

Thus, each trunk is characterized by 100 BSR values. In order to filter outliers and obtain smoother value sets, an averaging window was applied to each set. Moreover, for each patient, the angle values were normalized between $-100^{\circ}$ and $100^{\circ}$ to compensate for differences between the patients in term of severity.

\subsection{Classification}

We used support vector machines (SVMs) for the classification task. SVMs are particular linear classifiers that are based on the margin-maximization principle [10]. They are powerful classifiers which have been used successfully in many pattern recognition problems, and have also been shown to perform well in biometrics recognition applications [11].

Considering a binary classification problem with training data $\left\{\left(\mathrm{x}_{1}, \mathrm{y}_{1}\right), \ldots,\left(\mathrm{x}_{\ell}, \mathrm{y}_{\ell}\right)\right\}$, the SVM attempts to find the hyperplane $\langle\mathrm{w}, \mathrm{b}\rangle$ that maximizes the margin with minimum error:

$$
\min _{\mathrm{w}, \mathrm{b}, \mathrm{\xi}}\left(\frac{1}{2} \mathrm{w}^{\prime} \mathrm{w}+\mathrm{C} \sum_{\mathrm{i}} \xi_{\mathrm{i}}\right)
$$

s.t. $\quad \mathrm{y}_{\mathrm{i}}\left[\mathrm{w}^{\prime} \varphi\left(\mathrm{x}_{\mathrm{i}}\right)+\mathrm{b}\right] \geq 1-\xi_{\mathrm{i}} \quad$ and $\quad \xi_{\mathrm{i}} \geq 0 \quad \forall \mathrm{i}$ where $\mathbf{w}$ ' denotes the transpose of $\mathbf{w}, \varphi$ is the mapping function used implicitly via the kernel function $\mathrm{k}\left(\mathrm{x}_{\mathrm{i}}, \mathrm{x}_{\mathrm{j}}\right)=\varphi\left(\mathrm{x}_{\mathrm{i}}\right) \cdot \varphi\left(\mathrm{x}_{\mathrm{j}}\right)$ for non linear problems, $\mathrm{C}$ is used to balance the trade-off between maximizing the margin and minimizing the training error and $\xi$ is the slack variable that quantifies the SVM error. In the primal form, the Lagrangian of this problem is:

$$
\begin{aligned}
& \mathrm{L}=\frac{1}{2} \mathrm{w}^{\prime} \mathrm{w}+\mathrm{C} \sum_{\mathrm{i}} \xi_{\mathrm{i}}-\sum_{\mathrm{i}} \alpha_{\mathrm{i}}\left\{\mathrm{y}_{\mathrm{i}}\left[\mathrm{w}^{\prime} \varphi\left(\mathrm{x}_{\mathrm{i}}\right)+\mathrm{b}\right]-1+\xi_{\mathrm{i}}\right\} \\
& -\sum_{\mathrm{i}} \lambda_{\mathrm{i}} \xi_{\mathrm{i}}
\end{aligned}
$$

with the Lagrange multipliers $\alpha_{i} \geq 0$ and $\lambda_{i} \geq 0$ for all $\mathbf{i}$.

After taking the conditions for optimality, we obtain the classifier:

$$
f(x)=\operatorname{sign}\left[w^{\prime} \varphi(x)+b\right]=\operatorname{sign}\left[\sum_{i} \alpha_{i} y_{i} k\left(x_{i}, x\right)+b\right]
$$

with $\alpha$ solution of :

$$
\begin{aligned}
& \max _{\alpha}\left(\sum_{i} \alpha_{i}-\frac{1}{2} \sum_{i, j} \alpha_{i} \alpha_{j} y_{i} y_{j} k\left(x_{i}, x\right)\right) \\
& \text { s.t. } \sum_{i} \alpha_{i} y_{i}=0 \text { and } 0 \leq \alpha_{i} \leq C, \forall i
\end{aligned}
$$

The threshold $\mathbf{b}$ is computed by averaging:

$$
\mathrm{b}=\mathrm{y}_{\mathrm{j}}-\sum_{\mathrm{i}} \alpha_{\mathrm{i}} \mathrm{y}_{\mathrm{i}} \mathrm{k}\left(\mathrm{x}_{\mathrm{i}}, \mathrm{x}\right)
$$

over all support vectors $\mathrm{x}_{\mathrm{j}}\left(\alpha_{\mathrm{j}}>0\right)$.

Specifically for our application, we trained three SVMs using the one-against-all strategy with a radial basis function (RBF) kernel.

\subsection{Evaluation method}

Considering the size of our dataset $(\mathrm{N}=97)$ and the number of classes, we had no choice but to consider the same dataset for the classifier's training and testing. Among the various techniques for evaluating a classifier, we selected the leaveone-out cross validation method (LOOCV) because it is almost unbiased and its error should be relatively informative about the generalization error of the classifier [12]. The LOOCV algorithm considers a single observation from the original dataset as testing data and the remaining observations as training data. This procedure is repeated such that each observation is considered once as testing data. Finally, the classification error rate corresponds to the number of misclassifications divided by the number of observations.

\section{RESULTS}

On average, the scoliosis curve type was correctly predicted from measurements made on the trunk's surface in $72.2 \%$ of cases. This result shows that analysis of the trunk surface for scoliosis classification is feasible and promising.

As the confusion matrix shows (Table II), the best prediction rate is obtained for the third class, corresponding to the lumbar major curves. The maximal confusion was noted between classes 1 and 2. In fact, as illustrated in Fig. 3 , the mean BSR values of those two classes are quite similar and thus less distinct from one another compared to 
Table II - Confusion matrix

\begin{tabular}{|c|c|c|c|c|}
\hline & \multicolumn{3}{|c|}{ Target Class } \\
\hline & & 1 & 2 & 3 \\
\hline \multirow{3}{*}{$\begin{array}{l}\vec{J} \\
\frac{d}{0} \\
: \overrightarrow{0} \\
0 \\
0 \\
0\end{array}$} & 1 & 16 & 9 & 1 \\
\hline & 2 & 11 & 31 & 3 \\
\hline & 3 & 1 & 2 & 23 \\
\hline \multicolumn{2}{|c|}{$\begin{array}{l}\text { Prediction } \\
\text { rate per class }\end{array}$} & $57,1 \%$ & $73,8 \%$ & $85,2 \%$ \\
\hline
\end{tabular}

the mean values of the third class. Moreover, the variability between the observations within Class 2 was higher than for the remaining classes. This may be due to the fact that double and triple curves were considered as a single class.

\section{DISCUSSION}

In this work, we consider the BSR as the only clinical index to describe the trunk deformity. This choice is based on the clinical observation that the rib hump generally appears on the convex side of each spinal curve. Our results show that the BSR measured on 100 cross-sections of the trunk is a promising feature for this classification problem. However, even though the mean BSR values of each class seem quite distinct from each other, there is a high variability between patients of a same class.

To reduce the misclassification rate, we could consider adding more features of the trunk deformity. In the Lenke classification, a significant characteristic is the spinal flexibility evaluated on side-bending radiographs. Currently, we are working on a method, based exclusively on the topographic acquisition of the trunk, to assess the flexibility of the trunk. Including such a measure as a feature in our problem could increase the prediction rate.

Another way to improve the curve type prediction rate is to include more observations, especially taking into account the variability between patients of a given class. Moreover, a larger training dataset would allow us to consider more classes. Indeed, with a cohort of 97 patients, we focused on a high-level classification composed of 3 basic curve types, but with more data, we could aim at a hierarchical classification by adding a second level of finer resolution.

\section{CONCLUSION}

Current scoliosis classification systems rely on the evaluation of the spinal curve as seen in X-rays by clinicians. The data acquisition process is thus invasive. Moreover, those classifications do not take into account the general appearance of the trunk, which is the major concern for patients with scoliosis. In this paper, we propose a new system that predicts the scoliosis curve type from non invasive acquisitions of the trunk surface. The preliminary results for a high level classification are satisfactory and show the feasibility of our method.

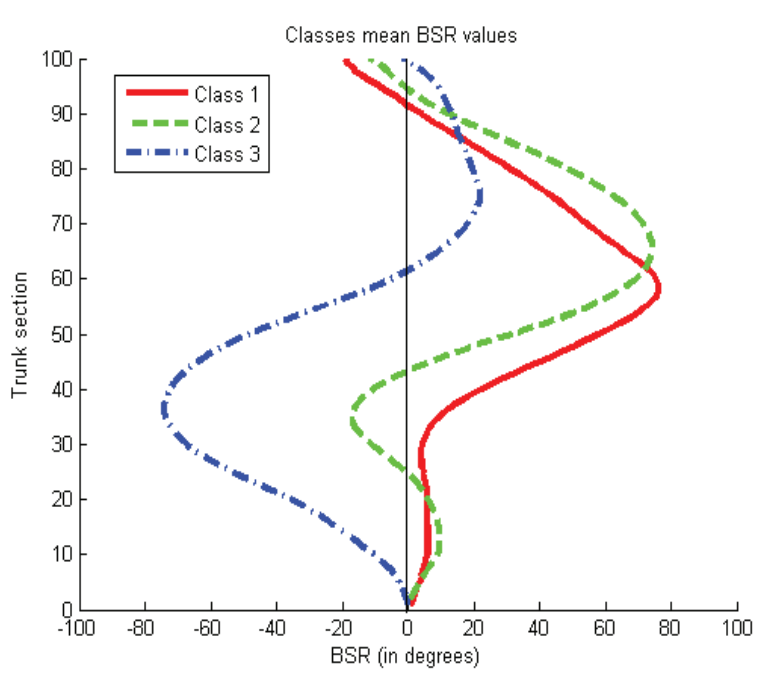

Fig. 3 - Mean BSR values for each section of the trunk, computed for each class.

\section{REFERENCES}

[1] M. M. Doody, J. E. Lonstein, M. Stovall et al., "Breast cancer mortality after diagnostic radiography: findings from the U.S. Scoliosis Cohort Study," Spine vol. 25, no. 16, pp. 2052-63., 2000. [2] L. Ramirez, N. G. Durdle, V. J. Raso et al., "A support vector machines classifier to assess the severity of idiopathic scoliosis from surface topography," IEEE Trans Inf Technol Biomed, vol. 10, no. 1, pp. 84-91, 2006.

[3] P. Ajemba, N. Durdle, D. Hill et al., "Classifying torso deformity in scoliosis using orthogonal maps of the torso," Medical and biological engineering and computing, vol. 45, pp. 575-584, 2007.

[4] I. A. Stokes, and M. S. Moreland, "Concordance of back surface asymmetry and spine shape in idiopathic scoliosis," Spine, vol. 14, no. 1, pp. 73-8, Jan, 1989.

[5] C. Bergeron, F. Cheriet, J. Ronsky et al., "Prediction of anterior scoliotic spinal curve from trunk surface using support vector regression," Engineering Applications of Artificial Intelligence, vol. 18, pp. 973-983, 2005.

[6] V. Pazos, F. Cheriet, L. Song et al., "Accuracy assessment of human trunk surface 3D reconstructions from an optical digitising system," Med Biol Eng Comput, vol. 43, no. 1, pp. 11-5, Jan, 2005. [7] V. Pazos, F. Cheriet, J. Danserau et al., "Reliability of trunk shape measurements based on 3-D surface reconstructions," Eur Spine J, vol. 16, no. 11, pp. 1882-91, Nov, 2007.

[8] L. G. Lenke, R. R. Betz, J. Harms et al., "Adolescent idiopathic scoliosis: a new classification to determine extent of spinal arthrodesis," J Bone Joint Surg Am, vol. 83-A, no. 8, pp. 1169-81, Aug, 2001.

[9] V. Pazos, F. Miled, P. Debanne et al., "Analysis of trunk external asymmetry in side-bending."

[10] V. N. Vapnik, Statistical learning theory, New York: John Wiley and Sons, 1998.

[11] M. M. Adankon, and M. Cheriet, "Support Vector machines," Encyclopedia of Biometrics, L. S. Z., ed., 2009.

[12] A. Luntz, and V. Brailovsky, "On estimation of characters obtained in statistical procedure of recognition "Technicheskaya Kibernetica, vol. 3, 1969. 